\title{
EVALUACIÓN DE LA SUSCEPTIBILIDAD DEL TERRENO POR MOVIMIENTOS EN MASA. ANÁLISIS CASO DE ESTUDIO: MICROCUENCA RÍO TIMBÍO - CAUCA.
}

\author{
EVALUATION OF THE SUSCEPTIBILITY OF THE TERRAIN FOR MOVEMENTS \\ MASS. CASE STUDY ANALYSIS: MICROBASIN RIVER TIMBÍO - CAUCA.
}

Dayan González-Quijano ${ }^{1,2, *}$; Elkin de Jesús Salcedo-Hurtado ${ }^{3}$

${ }^{1}$ Posgrado en Desarrollo Sustentable, Universidad del Valle. Ciudad Universitaria. Calle 100 No 13-
00. Cali, Colombia; dayan.gonzalez@correounivalle.edu.co - https://orcid.org/0000-0003-2438-7441
${ }^{2}$ Grupo de Estudios Ambientales. Universidad del Cauca. Calle 2 \# 1A- 25 Urbanización Caldas.
Popayán-Cauca. Colombia.
${ }^{3}$ Universidad del Valle, Departamento de Geografía. Ciudad Universitaria. Calle 100 No 13-00.
Cali, Colombia; elkin.salcedo@correounivalle.edu.co

*Correspondencia del Autor: Dayan González Quijano, correo electrónico: dayangonzalez651@gmail.com.

\section{RESUMEN}

En este artículo de investigación se evaluó la susceptibilidad del terreno ante movimientos en masa en la microcuenca del río Timbío - Cauca, mediante el uso de una metodología mixta basada en encuestas, entrevistas, estructuración de información cartográfica y el modelamiento espacial con el método bivariado. Los principales resultados de la investigación indican que los altos niveles de susceptibilidad se distribuyen por toda la microcuenca, además para el periodo comprendido entre 2012 - 2018 se registraron movimientos en masa que han ocasionado daños estructurales y ambientales. Los resultados obtenidos en la investigación se constituyen en un insumo base para fortalecer los procesos de planificación, direccionando así, el actuar de la comunidad y la institucionalidad frente a la gestión local del riesgo.

Palabras clave: Amenaza; gestión del riesgo de desastres; microcuenca; movimientos en masa; susceptibilidad.

Cómo citar:

González-Quijano, Dayan; Salcedo-Hurtado, Elkin de Jesús (2020). EVALUACIÓN DE LA SUSCEPTIBILI-DAD DEL TERRENO POR MOVIMIENTOS EN MASA. ANÁLISIS CASO DE ESTUDIO: MI-CROCUENCA RÍO TIMBÍO - CAUCA. Revista de Investigaciones Universidad del Quindio, vol. 32 (1), pp. 22-29. https://doi.org/10.33975/riuq.vol32n1.280 


\begin{abstract}
In this research paper assessed the susceptibility of the terrain before mass movements in the microbasin of the Timbío River - Cauca, using a mixed methodology based on surveys, interviews, structuring of cartographic information and spatial modeling with the bivariate method. The main results of the research indicate that high levels of susceptibility are distributed throughout the microbasin, and for the period between 2012-2018, mass movements were registered that have caused structural and environmental damage. the results obtained in the research constitute a base input to strengthen the planning processes, thus directing the actions of the community and institutions towards local risk management.
\end{abstract}

Keywords: Hazard; disaster risk management; microbasin; mass movements; susceptibility.

\section{INTRODUCCIÓN}

El mal manejo de los recursos naturales, están aumentando de forma considerable, causando graves repercusiones de tipo ambiental, social, cultural, político y económico; el ser humano ha ejercido un papel fundamental en la modificación de los elementos que conforman la superficie terrestre (Suarez, 1995), ejerciendo un alto grado de presión que afecta la sostenibilidad de los territorios. Los temas ambientales planteados en las cumbres mundiales se relacionan con el cambio climático, la contaminación del agua, la sequía, el deterioro de los suelos, las inundaciones y los movimientos en masa (Martínez, 2009).

En Colombia se ha presentado un aumento en la ocurrencia de eventos desastrosos relacionados con causas naturales, la intervención humana y el consecuente deterioro ambiental (Banco Mundial, 2012). Los fenómenos que han generado mayor número de víctimas a nivel nacional son los movimientos en masa (Banco Mundial, 2012), los cuales representan un peligro latente que puede considerarse como una amenaza para el desarrollo social y económico de una región (Cardona, 1993).

En el Departamento del Cauca, los movimientos en masa han afectado a 33 municipios y más de 105.960 personas (CDGRD, 2015). Estos fenómenos son ocasionados principalmente por actividades antrópicas, generando afectaciones en viviendas, poblaciones, infraestructura vial y acueductos (SGC, 2017). Además, estos eventos generan daños psicológicos que afectan el desarrollo de las actividades diarias de los habitantes de un determinado lugar.

Teniendo en cuenta lo mencionado anteriormente, el estudio de amenazas por movimientos en masa, se debe establecer como una prioridad para el análisis de la gestión del riesgo de desastres en Colombia, haciendo énfasis en la inversión para el conocimiento y la reducción del riesgo (MVCT-UNGRD, 2014). Por tanto, mediante el desarrollo de la presente investigación se evalúa la susceptibilidad del terreno ante movimientos en masa, mediante un trabajo técnico y participativo en la microcuenca del río Timbío, con el fin de generar un insumo que permita fortalecer y direccionar los procesos de gestión del riesgo a nivel local.

\section{MATERIALES Y METODOS}

\section{Área de estudio}

La microcuenca del río Timbío se encuentra ubicada en el Municipio de Sotará, en el Departamento del Cauca en medio de las cordilleras central y occidental, al Suroccidente de la República de Colombia (CMGRD, 2015). Con coordenadas ( $2^{\circ} 18^{\prime} 18.50^{\prime \prime}$ Norte, $76^{\circ} 35^{\prime} 35.62^{\prime \prime}$ Oeste), se encuentra dentro de la cuenca del río Cauca, nace en el cerro las Estrellas a 2200 msnm y cuenta con una extensión de 686 hectáreas (Alcaldia Municipal, 2000). Así mismo, es la principal fuente abastecedora del acueducto municipal de Timbío.

Esta zona se caracteriza por poseer un clima templado húmedo en relieves fuertemente ondulados, con pendientes de $12-25 \%$ de inclinación. De igual forma, son suelos moderadamente profun- 
dos, bien drenados con texturas moderadamente gruesas a finas (IGAC, 2009). El uso del suelo está dedicado a la agricultura, la ganadería y la extracción de material de arrastre como arena y lodo, además de la extracción de madera cerca de los cauces de los ríos (CMGRD, 2015).

La microcuenca está conformada por las veredas La Estrella y El Salado, se identifican alrededor de 14 viviendas que por lo general son de composición unifamiliar, pertenecientes a comunidades campesinas e indígenas que convergen en el mismo territorio.

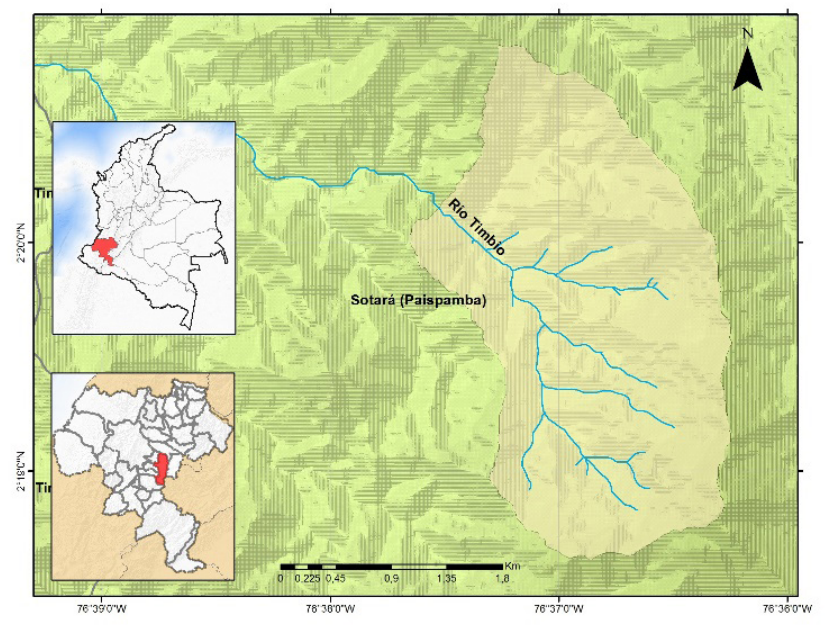

Figura 1. Mapa de ubicación de la microcuenca del Río Timbío.

Fuente: Elaboración propia

El análisis se desarrolla en dos etapas: i) Construcción del inventario de movimientos en masa mediante un trabajo participativo con los habitantes de la microcuenca del Río Timbío. ii) Modelamiento espacial de la susceptibilidad del terreno ante movimientos en masa mediante el método bivariado. A continuación, se menciona a detalle cada una de las etapas:

\section{Etapa 1. Construcción del inventario de mo- vimientos en masa mediante un trabajo parti- cipativo con los habitantes de la microcuenca del río Timbío}

Debido a la falta de información histórica de los de los movimientos en masa ocurridos en la zona de estudio, se hace necesario un trabajo participativo basado en encuestas y entrevistas; las herramientas aplicadas están basadas en los trabajos desarrollados por Sampieri, Fernández, \& Baptista, 2014 y Salcedo, 2018. La encuesta denominada "Percepción de los movimientos en masa en su territorio" se aplicó a 14 personas integrantes de cada una de las familias que habitan en la microcuenca. Las encuestas se realizaron con personas mayores de edad que tienen diferentes niveles de escolaridad. Posteriormente se realizaron 5 entrevistas a personas claves (líderes y personas afectadas por movimientos en masa) que habitan en la microcuenca y son conocedores de las dinámicas ambientales y sociales.

Finalmente, la información obtenida en las entrevistas y encuestas es procesada en Excel, corroborada en campo y espacializada en un SIG, generando el inventario de movimientos en masa.

\section{Etapa 2. Modelamiento espacial de la suscepti- bilidad del terreno ante movimientos en masa mediante el método bivariado}

El análisis bivariado de la susceptibilidad del terreno se basó en la metodología utilizada por (Westen, Montoya, \& Vargas, 1996) y aplicada en un contexto regional y local por (Alvarado F, Medina B, Sandoval R, \& Garcia N, 2016) y (UNIVALLE \& CVC, 2018). Es importante mencionar que el procesamiento del modelo espacial se realiza en la plataforma ArcGis y las variables a tener en cuenta (pendientes, suelos, coberturas vegetales), cumplen con un alto nivel de detalle, a excepción de la variable suelos que presenta una escala mayor de detalle. La información base es suministrada por entidades como el Instituto Geográfico Agustín Codazzi (IGAC) y el Servicio geológico de los Estados Unidos (USGS).

A continuación, se menciona la metodología para la estructuración de las variables:

\section{- Coberturas vegetales}

El análisis de coberturas vegetales se basó en un mosaico de imágenes satelitales Sentinel en un 
periodo de tiempo comprendido entre los años 2016 y 2018, mediante una clasificación supervisada en la plataforma ArcGis 10.3; el procesamiento se ajustó teniendo en cuenta los puntos de muestreo levantados en campo y talleres de cartografía social con el fin de generar un nivel de precisión mayor para el análisis de cobertura del suelo.

\section{- Pendientes}

El mapa de pendientes de la zona de estudio, se generó a partir de un modelo de elevación digital (DEM) con una resolución de 12,5 metros por pixel, los rangos de pendiente asignados corresponden a la clasificación propuesta por (Zuidam, 1989), enfocada en el análisis de movimientos en masa.

\section{- Suelos}

La capa es estructurada a partir del estudio de Suelos del Cauca del año 2009, elaborado por el Instituto Geográfico Agustín Codazzi (IGAC).

\section{Modelamiento espacial}

El modelamiento de la susceptibilidad mediante el método bivariado, consiste en el análisis de la cantidad de movimientos en masa para cada una de las unidades de los factores que influyen en su ocurrencia, teniendo en cuenta una variable dependiente (movimientos en masa) y una independiente (resto de variables), además el análisis se puede realizar por la combinación de parámetros (Alvarado F et al., 2016). Es decir, la ponderación estadística de factores permite asignarles un peso a las variables teniendo en cuenta la densidad de los movimientos en masa para cada parámetro (Westen et al., 1996).

Por ello la probabilidad (P), de la ocurrencia del evento en condiciones similares (A) puede ser modificada con valores (B) que son los factores causantes. Al integrar los valores se determina una probabilidad posterior $\mathrm{P}(\mathrm{A} / \mathrm{B})$ que indica que un evento (A) ocurra por un factor causante (B)
(UNIVALLE \& CVC, 2018). Así como se puede observar en la siguiente formula:

$$
P=\frac{A}{B}=+\frac{P(A) * P\left(\frac{B}{A}\right)}{P(B)}
$$

Posteriormente se calculan los pesos para las tres variables indicando una correlación o ausencia entre los factores causantes. Para evaluar la combinación de los factores se tiene en cuenta el modelo de elevación digital de 12,5 metros y el número de celdas donde se presentan los eventos. Siendo $(\mathrm{W})$ el peso de un parámetro especifico, (DC) la densidad de los movimientos en masa para cada parámetro, (DM) la densidad de eventos para el área de estudio, (AD) el área con presencia de movimientos en masa y (AT) el área total del parámetro considerado (Westen et al., 1996), como se puede observar en la siguiente formula:

$$
w=\ln =\frac{D C}{D M}=\left(\frac{\left(\frac{A D}{A T}\right)}{\left(\sum \frac{A D}{A T}\right)}\right)
$$

En este sentido, para la estandarización de la densidad de los movimientos en masa se relaciona las densidades de cada parámetro con la densidad general para la zona de estudio. De igual manera se calcula el logaritmo natural, con el fin de asignar un peso negativo cuando la densidad de movimientos es baja y positivo cuando sea alta (Westen et al., 1996).

A continuación, se relacionan los pesos asignados a cada parámetro:

Tabla 1. Pesos de cada variable

\begin{tabular}{|c|c|}
\hline Variables & Rango en pesos \\
\hline Pendiente & $-19.2-7.62$ \\
\hline Suelo & $-0.3-3.11$ \\
\hline Coberturas del suelo & $-1.7-10.23$ \\
\hline
\end{tabular}

Finalmente, con el cálculo de los pesos de cada variable espacializada se hace una sumatoria, generando el índice de susceptibilidad por movimientos en masa (LSI), como se puede ver en la 
siguiente ecuación:

$$
L S I=w P+w C+w S
$$

Con los valores de susceptibilidad obtenidos se determinan cinco categorías de análisis: "Muy alta", "Alta", "Media", "Baja" y "Muy baja", teniendo en cuenta que cuando los valores son negativos se clasifican dentro de rangos de susceptibilidad bajo y medio, mientras que los valores positivos se distribuyen en rangos altos.

\section{RESULTADOS Y DISCUSIÓN}

\section{Inventario de movimientos en masa}

El procesamiento de las encuestas y entrevistas realizadas con los habitantes de la microcuenca del río Timbío, arrojan como resultado que existe un reconocimiento de que los movimientos en masa representan una amenaza latente para el desarrollo de la zona. Este reconocimiento parte de una experiencia traumática que ocasiono repercusiones psicológicas y culturales a las personas afectadas. Dicho evento se presentó en marzo de 2012 en la parte alta de la microcuenca del Río
Timbío que genero grandes afectaciones en 5 viviendas de constitución unifamiliar, que fueron evacuadas tras el suceso. En las entrevistas los habitantes de la microcuenca manifiestan que la ayuda del gobierno local, solamente beneficio a una de las familias afectadas.

En los relatos de la comunidad también se manifiesta que los movimientos en masa se presentan en época de invierno en terrenos con altas pendientes y son ocasionados por las actividades antrópicas que se desarrollan en la zona. Sumado a ello, en inmediaciones del cauce del río, se presentan movimientos en masa que afectan en menor proporción la calidad del agua que es suministrada al acueducto que abastece la cabecera municipal de Timbío-Cauca.

Teniendo en cuenta la memoria histórica de los habitantes de microcuenca del río Timbío, se construyó un inventario de movimientos en masa en un periodo de tiempo comprendido entre los años 2012 y 2018, el cual cuenta con 5 registros. A continuación, se relaciona el inventario por movimientos en masa (Ver tabla $\mathrm{N}^{\mathrm{o}} 2$ ).

\begin{tabular}{|ccc|}
\hline & Inventario de Movimientos en masa \\
\hline Fecha de ocurrencia & \multicolumn{3}{c|}{ Coordenadas } \\
$1 / 03 / 2012$ & $2^{\circ} 18^{\prime} 48.82^{\prime \prime} \mathrm{N}$ & $76^{\circ} 36^{\prime} 57.544^{\prime \prime}$ \\
$27 / 04 / 2012$ & $2^{\circ} 18^{\prime} 59.17^{\prime \prime} \mathrm{N}$ & $76^{\circ} 37^{\prime} 03.233^{\prime \prime}$ \\
$1 / 05 / 2015$ & $2^{\circ} 19^{\prime} 48.38^{\prime \prime} \mathrm{N}$ & $76^{\circ} 36^{\prime} 57.37^{\prime}$ \\
$12 / 10 / 2018$ & $2^{\circ} 19^{\prime} 18.86^{\prime \prime} \mathrm{N}$ & $76^{\circ} 36^{\prime} 49.08^{\prime \prime}$ \\
$12 / 10 / 2018$ & $2^{\circ} 20^{\prime} 14.84^{\prime \prime} \mathrm{N}$ & $76^{\circ} 37^{\prime} 36.36^{\prime \prime}$ \\
\hline
\end{tabular}

Tabla 2. Inventario de movimientos en masa

Modelamiento espacial de susceptibilidad del terreno

\section{Descripción de variables y método bivariado}

La estructuración de información cartográfica arroja como resultado que en las Coberturas vegetales de la microcuenca del Río Timbío, predominan los bosques de galería o riparío con un $43,29 \%$, seguido por los bosques secundarios con un con un $21.89 \%$, indicando que los niveles de conservación son altos, situación que favorece la regulación del recurso hídrico. De forma inversa, los suelos desnudos se presentan en menor proporción con un 5,33\%, cobertura asociada a suelos donde existieron algún tipo de cultivos o son suelos removidos por deslizamientos.

Es importante mencionar que en la microcuenca se presentan actividades como el sobrepastoreo del ganado de doble propósito, la ampliación de la frontera agrícola y algunos cultivos de pan co- 
ger, estas actividades agrícolas y pecuarias se dan a pequeña escala, afectando el equilibrio ecológico de la microcuenca y la estabilidad de los terrenos, sin embargo se reconoce que las actividades son desarrolladas por campesinos e indígenas residentes en la zona y están basadas en prácticas ancestrales que en la mayoría de los casos son amigables con el medio ambiente. Por otro lado, en la parte alta de la microcuenca se realiza extracción maderera por la empresa Smurfit Kappa o cartón de Colombia, la cual tiene grandes plantaciones en la zona que perjudican la sostenibilidad de los recursos naturales.

Los valores de pendiente predominantes en la zona de estudio, corresponden a un rango abrup- to $\left(16\right.$ a $\left.35^{\circ}\right)$ con un $67.1 \%$, estas pendientes se caracterizan por ser inclinadas y propensas al desarrollo de procesos erosivos; Posteriormente, se encuentran las pendientes moderadamente abruptas $\left(8-16^{\circ}\right)$, que se caracterizan por ser susceptibles a deslizamientos.

Los suelos predominantes en la microcuenca, están representados por la unidad cartográfica LLAf3, conformada por cenizas volcánicas sobre rocas ígneas. Este tipo de material, se constituye por suelos bien drenados de texturas franco arenosas, ubicados sobre un relieve moderadamente escarpado que facilita la erosión moderada y severa, generando así, movimientos en masa (IGAC, 2009).

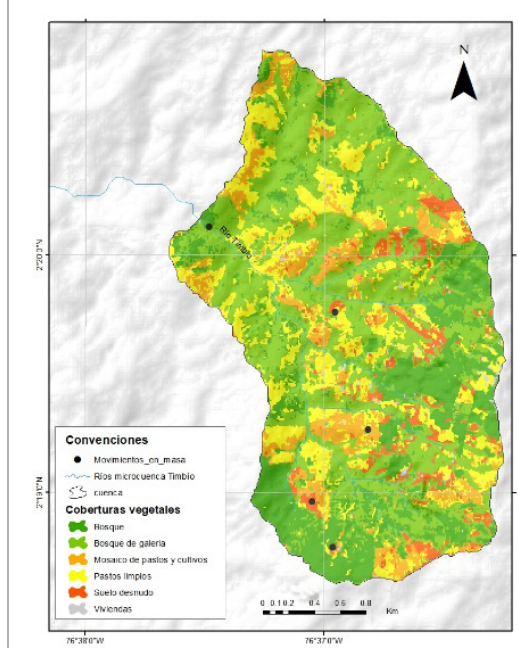

Figura $N^{0}$ 2. Distribución espacial de coberturas vegetales.

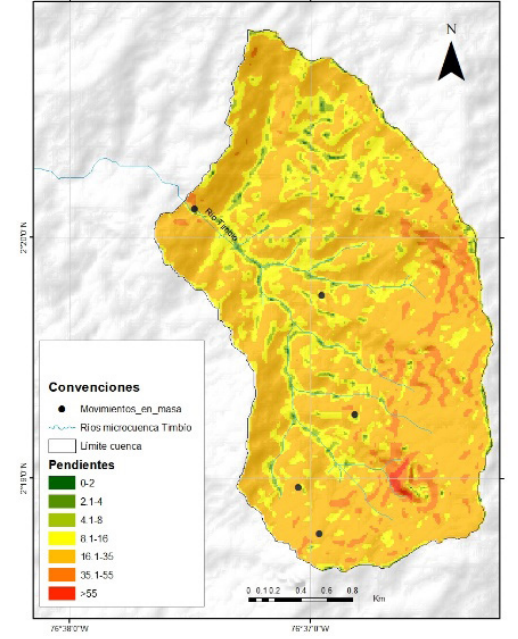

Figura $\mathbf{N}^{0}$ 3. Distribución espacial de Pendientes.

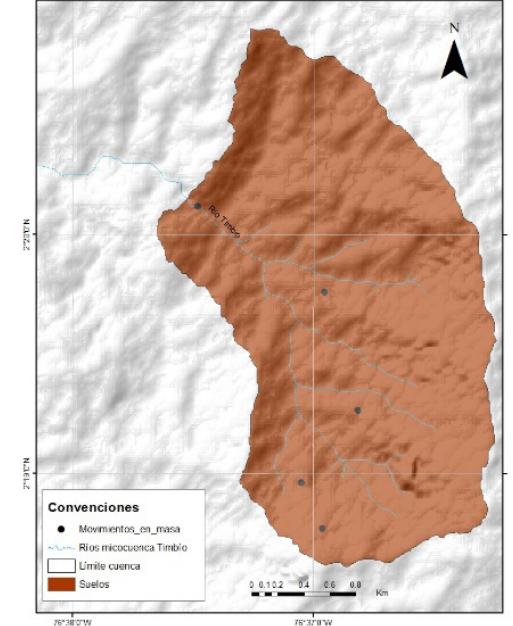

Figura $\mathbf{N}^{0}$ 4. Distribución espacial de suelos.
Al correr el modelo espacial con el método bivariado, se obtuvo como resultado que en la microcuenca del río Timbío predomina una susceptibilidad "Baja" con un 43.3\%, la cual se encuentra distribuida espacialmente en la zona suroriente, donde las pendientes son pronunciadas pero los procesos de conservación del ecosistema son altos. Seguido por la categoría de susceptibilidad "Alta" con un 40,2 \%; distribuida en la zonas norte y sur occidente; es importante mencionar que en esta categoría se encuentran ubicadas 5 viviendas distribuidas espacialmente en la parte media y baja de la microcuenca que pueden verse afectadas por la ocurrencia de un movimiento en masa. Paralelamente, se encuentra la categoría de susceptibilidad "Muy alta" con un valor de 12.7 $\%$ y se encuentra distribuida por toda la microcuenca en pequeñas áreas, en esta categoría se puede observar la ubicación de un movimiento en masa activo en la zona alta que puede presentar recurrencia en época de invierno (ver mapa $\mathrm{N}^{\circ} 4$ $y$ grafico $\mathrm{N}^{\circ} 1$ ). 


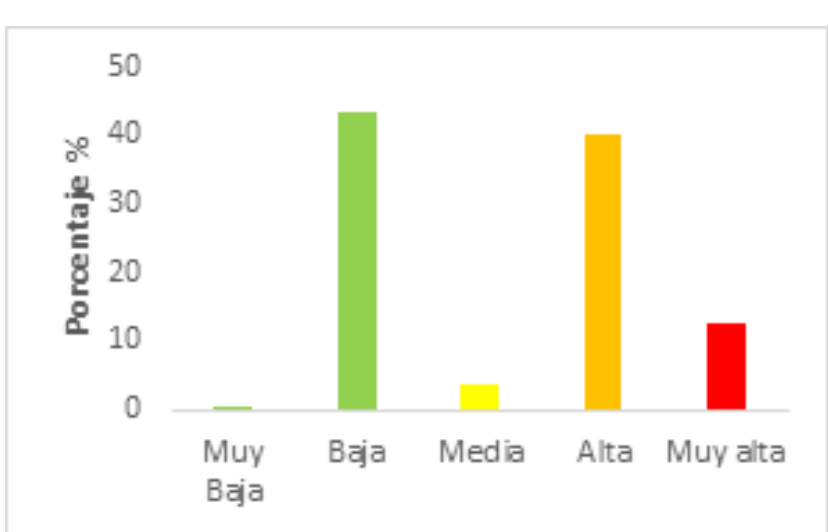

Rangos de susceptibilidad

Gráfico $\mathbf{N}^{\mathbf{0}}$ 1. Porcentaje de susceptibilidad

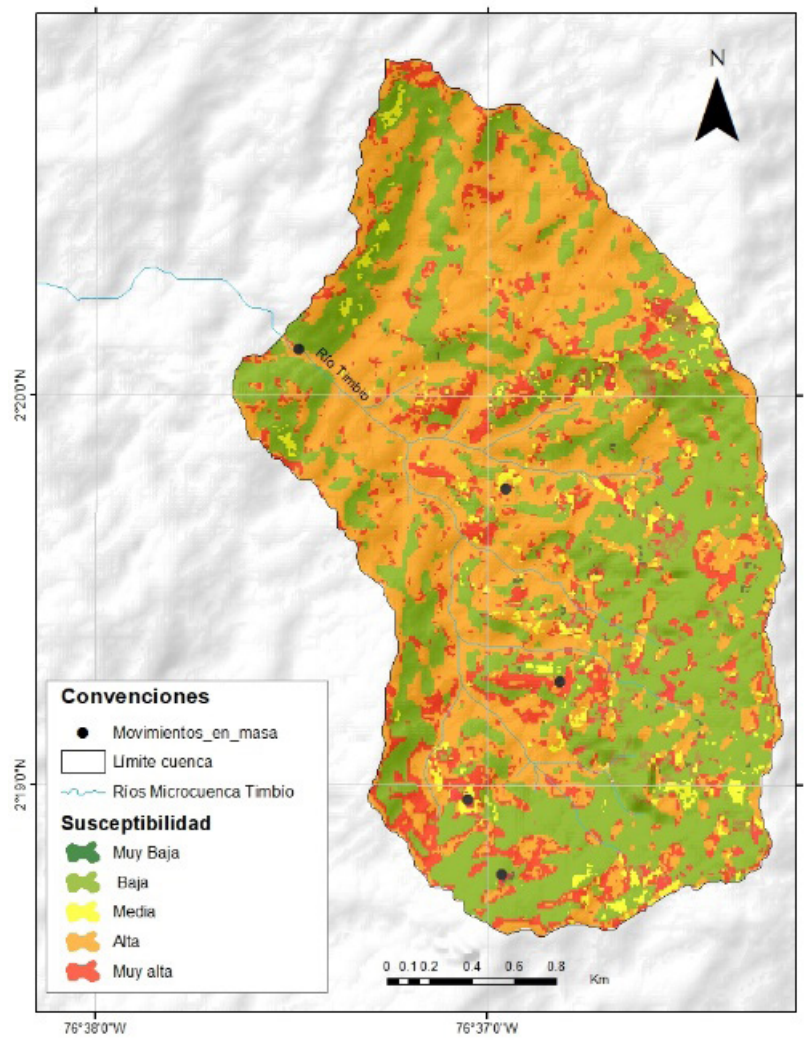

Figura $\mathbf{N}^{\mathbf{0}}$ 5. Distribución espacial de la susceptibilidad

La validación del modelo se hace mediante la revisión de los puntos de muestreo (movimientos en masa), los cuales se encuentran ubicados en su gran mayoría en zonas de susceptibilidad alta y muy alta; indicando que se presentan patrones similares al correlacionar la información de las tres variables estudiadas.

\section{CONCLUSIONES}

- En la zona de estudio no existe un registro oficial de los movimientos en masa, por ello, los recuentos y la memoria histórica de los habitantes arrojan resultados valiosos que permiten hacer una reconstrucción de los movimientos en masa ocurridos.

- Los procesos de globalización y constante cambio, implican modificaciones en las coberturas vegetales que alteran la estabilidad de los terrenos y generan altos índices de susceptibilidad a movimientos en masa.

- El método bivariado aplicado en zonas específicas, debe contar con registros históricos y cartografía a un alto nivel de detalle, con el fin de correlacionar las características de las variables con los puntos de muestreo.

- Los resultados de la investigación se constituyen en un insumo fundamental para la actualización de los Planes de Gestión del Riesgo y los Planes de Manejo y Ordenamiento de Cuencas Hidrográficas, ampliando así el que hacer de la institucionalidad frente a eventos que pueden generar grandes afectaciones en los territorios.

\section{AGRADECIMIENTOS}

Agradecemos al proyecto Vulnerabilidad y riesgo en Sistemas de Abastecimiento en el Cauca (AQUARISC) por su financiación en la investigación, al Observatorio Sismológico del Sur Occidente Colombiano (OSSO) de la Universidad del Valle y al Grupo de Estudios Ambientales (GEA) de la Universidad del Cauca por el direccionamiento técnico durante el modelamiento espacial. Finalmente agradecemos a la comunidad de la microcuenca del Río Timbío, por su valiosa colaboración durante el desarrollo de la investigación. 


\section{BIBLIOGRAFÍA}

1. Alcaldia Municipal. (2000). Plan basico de ordenamiento territorial del Municipio de Timbío, Diagnostico territorial, p. 92. Timbío, Cauca.

2. Alvarado F, C., Medina B, E., Sandoval R, J., \& Garcia N, J. (2016). Zonificación de amenaza por movimientos en masa aplicando un método bivariado y un proceso heurístico. Caso aplicado a la cuenca del Río Combeima, Ibagué-Tolima-Colombia http://doi.org/10.13140/RG.2.2.20103.19361

3. Banco Mundial. (2012). Analisis de la gestión del riesgo de desastres en Colombia: Un aporte para la construcción de políticas publicas. p. 438.

4. Cardona, O. D. (1993). Evaluación de la amenaza, la vulnerabilidad y el riesgo. Los Desastres No Son Naturales, pp. 45-65.

5. CMGRD. (2015). Plan municipal de gestión del riesgo de desastres, Timbío- Cauca. Timbío, Cauca.

6. IGAC. (2009). Clasificación de las tierras por capacidad de uso. In Estudio general de suelos y zonificación de tierras, Departamento del Cauca. Escala 1:100.000 . Bogotá- Colombia.

7. Martínez, M. (2009). Los geógrafos y la teoría de riesgos y desastres ambientales. Perspectiva Geográfica, Vol. 14. Tunja- Colombia.

8. MVCT-UNGRD. (2014). Herramienta metodológica para la formulación de programas de gestión del riesgo de desastres en los servicios acueducto, alcantarillado y aseo. p. 68. Bogotá- Colombia.

9. Salcedo, E. (2018). Matriz de amenazas e impactos. Santiago de Cali- Colombia.

10. Sampieri, R., Fernández, C., \& Baptista, M. del P. (2014). Metodologia de la investigación. México.

11. SGC. (2017). Las amenazas por movimientos en masa: Una visión a escala 1:100.000. p. 321. BogotáColombia.

12. Suarez, J. (1995). Procesos de origen Antrópico. In Deslizamientos. p. 335-354.

13. UNIVALLE, \& CVC. (2018). Zonificación de la amenaza por Movimientos en Masa de la zona urbana del Municipio de Versalles. Cali- Colombia.

14. Westen, V., Montoya, L., \& Vargas, R. (1996). Aplicación del SIG para la Evaluación de la Amenaza y el Riesgo: Tegucigalpa, Honduras. p. 1-14.

15. Zuidam, V. (1989). Aerial photo-interpretation in terrain analysis and geomorphologic mapping: The Hague, Smits, The Netherlands. 